\title{
Efeitos da realidade virtual na qualidade de vida em indivíduos parkinsonianos
}

\author{
Effects of virtual reality on quality of life in parkinsonian \\ individuals
}

\author{
Efectos de larealidad virtual sobre la calidad de vida de las \\ personas parkinsonianas
}

\author{
Ana Sandrina da Mota Gantuss ${ }^{1}$, Karoline Chagas da Silva ${ }^{1}$, Nathália \\ Katlem de Araújo Alho' ${ }^{1}$, José Alexandre Silva Júnior ${ }^{2}$
}

1.Fisioterapeuta. Instituto Esperança de Ensino Superior- IESPES, Santarém-PA, Brasil.

2.Fisioterapeuta, Mestre em Engenharia Biomédica, docente do curso de Fisioterapia do Instituto Esperança de Ensino Superior-IESPES, Santarém-PA, Brasil.

\begin{abstract}
Resumo
Introdução. A doença de Parkinson (DP) é uma afecção neurodegenerativa de neurônios dopaminérgicos da substância nigra dos núcleos da base, esta degeneração resulta em uma diminuição da produção de dopamina. Em indivíduos com DP, a diminuição dopaminérgica leva ao surgimento de sinais como: bradicinesia, tremor de repouso, rigidez e instabilidade postural. Objetivo. O presente estudo tem como objetivo explanar e estabelecer conceitos, técnicas e resultados em relação a utilização da realidade virtual (RV) no tratamento de Parkinson, estruturando-se em estudos com bases em evidências cientificas. Método. Tratase de uma pesquisa de revisão, com levantamento de dados nas bases Google Acadêmico, Scielo, Pubmed e Web of Science. Resultados. Após a busca e escolhas dos artigos, sete estudos foram incluídos, sendo eles um ensaio clínico, ensaio clínico randomizado, ensaio clínico randomizado controlado, estudo piloto, ensaio clínico duplo-cego randomizado, estudo observacional prospectivo e ensaio clínico não controlado. Verificou-se que a maioria dos estudos analisados se relacionava a equilíbrio (28,57\%), seguido de cognição $(14,28 \%)$, congelamento de marcha $(28,57 \%)$ e bem-estar emocional $(28,57 \%)$. Todos com objetivos voltado para a melhora da QV desses indivíduos. Conclusão. A RV empregada como uma ferramenta de reabilitação em pacientes com DP se mostrou eficaz na melhora da QV deles. No entanto, se faz necessário a produção de estudos científicos que enfoquem a durabilidade dos efeitos causados pela aplicação da RV na sintomatologia da DP.
\end{abstract}

Unitermos. Realidade Virtual; Parkinson; Qualidade de vida; Fisioterapia

\begin{abstract}
Introduction. Parkinson's disease (PD) is a neurodegenerative disorder of dopaminergic neurons of the nigra substance in the nuclei of the base, this degeneration results in a decrease in dopamine production. On request with $\mathrm{PD}$, the dopaminergic decrease leads to the appearance of signs such as: bradykinesia, rest tremor, stiffness, and postural instability. Objective. This study aims to explain and establish concepts, techniques, and results in relation to the use of virtual reality (VR) in the treatment of Parkinson's, structuring itself in studies based on scientific evidence. Method. This is a review with data collection in the Google Scholar, Scielo, Pubmed, and Web of Science databases. Results. After searching and choosing the articles, seven studies were included, including a clinical trial, randomized clinical trial, randomized controlled clinical trial, pilot study, randomized double-blind clinical trial, prospective observational study, and uncontrolled clinical trial. It was found that most of the relative studies were related to balance $(28.57 \%)$, followed by cognition $(14.28 \%)$, gait freeze $(28.57 \%)$ and emotional well-being $(28.57 \%)$. All with objectives aimed at improving the QOL, necessary. Conclusion. The VR used as a rehabilitation tool in PD patients proved to be effective in improving their QOL. However, it is necessary to produce
\end{abstract}


scientific studies that focus on the durability of the effects caused by the application of VR in the symptoms of PD.

Keywords. Virtual Reality; Parkinson; Quality of life, Physical Therapy

\section{Resumen}

Introducción. La enfermedad de Parkinson (EP) es untrastorno neurodegenerativo de lasneuronas dopaminérgicas de lasustancianigraenlos núcleos de la base, esta degeneración da como resultado una disminuciónenlaproducción de dopamina. A pedido de la EP, ladisminución dopaminérgica conduce a laaparición de signos como: bradicinesia, temblor de reposo, rigidez e inestabilidad postural. Objetivo. Este estudiotiene como objetivo explicar y establecer conceptos, técnicas y resultados enrelación al uso de larealidad virtual (RV) eneltratamientodel Parkinson, estructurándoseenestudiosbasadosenevidencia científica. Método. Se trata de una investigación de revisión, conrecopilación de datosenlas bases de datos de Google Scholar, Scielo, Pubmed y Web of Science. Resultados.Después de buscar y elegirlos artículos, se incluyeronsieteestudios, incluidounensayo clínico, ensayo clínico aleatorizado, ensayo clínico controlado aleatorizado, estudio piloto, ensayo clínico aleatorizado doble ciego, estudio observacional prospectivo y ensayo clínico no controlado. Se encontró que lamayoría de losestudios relativos estaban relacionados conelequilibrio $(28,57 \%)$, seguido de lacognición $(14,28 \%)$, la marcha congelada $(28,57 \%)$ y elbienestar emocional (28,57\%). Todo ellocon objetivos encaminados a mejorarla CV, necesarios. Conclusión. La RV utilizada como herramienta de rehabilitaciónen pacientes con EP demostró ser eficaz para mejorarsucalidad de vida. Sin embargo, es necesarioproducirestudios científicos que se enfoquenenladurabilidad de losefectos provocados por laaplicación de la RV enlossíntomas de la EP.

Palabras clave. Realidad virtual; Parkinson; Calidad de vida; Fisioterapia

Trabalho realizado no Instituto Esperança de Ensino Superior- IESPES, Santarém-PA, Brasil.

\section{INTRODUÇÃO}

\section{A doença de Parkinson (DP) é uma afecção} neurodegenerativa de neurônios dopaminérgicos da substância nigra dos núcleos da base, esta degeneração resulta em uma diminuição da produção de dopamina. Em indivíduos com DP, a diminuição dopaminérgica leva ao surgimento de sinais como: bradicinesia, tremor de repouso, rigidez e instabilidade postural ${ }^{1}$. Além dos sintomas motores a doença também leva a problemas cognitivos, neuropsiquiátricos, comportamentais e sensoriais, distúrbios do sono e problemas relacionados à tomada de decisões, memória, depressão, ansiedade e 
percepção visual-espacial, as queixas mais preocupantes são as de quadros depressivos e queixas cognitivas, por serem fatores que contribuem para um pior prognostico na qualidade de vida (QV) desses pacientes².

Estudos recentes mostram que devido ao envelhecimento da população mundial, a estimativa esperada até o ano de 2020 era de mais de 40 milhões de $\operatorname{casos}^{3}$. O conjunto de etiologia que pode estar relacionado ao surgimento da DP destaca-se: fatores genéticos, toxinas ambientais, estresse oxidativo, anormalidades mitocondriais, entre outros. Os maiores números de casos apresentam-se na faixa etária dos 50 aos 70 anos, sendo este mais frequente no sexo masculino ${ }^{4}$.

A relação de sintomas motores e não motores, gera muitas vezes nesses individuos perda de independência, medo de quedas e inatividade, o que consequentemente resulta em isolamento social e aumento do risco de osteoporose ou doenças cardiovasculares ${ }^{5}$. Como resultado, esses individuos tendem a apresentar limitações de mobilidade, comunicação e motoras para as atividades de vida diária, o que esta intimamente relacionada à pior percepção geral ${ }^{6}$. A QV nessa população é especificamente atingida em suas atividades laborais sendo diretamente ligado ao nível e estágio da doença, onde a QV é dita como multidimensional de uma avaliação subjetiva de cada indivíduo ${ }^{7}$.

Em virtude dos sinais e sintomas a abordagem terapêutica tem o enfoque na manutenção ou recuperação 
da QV, assim englobando o termo "qualidade de vida relacionada à saúde" (QVRS). Dessa maneira cada paciente terá um protocolo individualizado conforme as suas necessidades frente a multidimensionalidade da QV7. Sendo assim, a terapêutica constantemente usada é a estimulação de ativação da neuroplasticidade, também conhecida como adaptação, habituação e substituição, onde a planos terapêuticos comumente utilizados são: atividades em dupla tarefa, treino de equilíbrio convencional, motricidade geral e reabilitação vestibular ${ }^{8}$.

$\mathrm{Na}$ última década, a realidade virtual (RV) tomou grande espaço nos tratamentos de reabilitação para pacientes neurológicos, onde o paciente pode interagir com a simulação do ambiente através de vários canais sensoriais. O treinamento de RV pode ser aplicado usando dispositivos disponíveis no mercado (como, Wii com balance board) ou o protótipo desenvolvido pelos pesquisadores ${ }^{9}$. Alguns estudos propõem que os videogames são capazes de promover o treinamento motor-cognitivo integrado com o potencial de melhorar o equilíbrio, aprendizagem motora, cognição e independência nas atividades da vida diária dos indivíduos com DP ${ }^{10}$. Por meio destes recursos os pacientes tem a possibilidade de realizarem tarefas complexas (rápidas e grandes movimentos envolvendo todo o corpo, em vez de realizar o movimento em uma única articulação). Além disso, podem fornecer feedback auditivo e visual que pode contribuir para o desempenho físico ${ }^{11}$. 
Todos esses aspectos podem ser alcançados pela RV, visto que é um treino de percepção ambiental, o conflito sensorial gera um ganho no reflexo vestíbulo-ocular sendo capaz de recuperar ou reestabelecer manutenções e funções laborais ${ }^{12}$.

O presente estudo tem como objetivo explanar e estabelecer conceitos, técnicas e resultados em relação a utilização da realidade virtual no tratamento de Parkinson, estruturando-se em estudos com bases em evidências cientificas.

\section{MÉTODO}

Trata-se de uma revisão integrativa que consiste em uma investigação cientifica própria, onde reúne diversos resultados de pesquisas primarias proposta nesse estudo, sendo selecionados através de critérios relevantes ao tema ${ }^{13}$. Para dar continuidade a revisão, seguiu-se a pesquisa com o seguinte questionamento: Quais os efeitos da realidade virtual na qualidade de vida em indivíduos parkinsonianos?

Realizou-se as buscas nas seguintes bases de dados: Google acadêmico, Pubmed,Scientific Eletronic Libray Online (Scielo) e Web ofscience com os seguintes descritores: Realidade virtual (Reality virtual), Parkinson, Qualidade de vida (Qualidyoflife).

Como critérios de inclusão para busca houve delimitação de período, com artigos entre 2010 e 2020, sendo selecionados estudos em português e inglês. Estes 
deveriam conter assuntos relacionados ao uso da realidade virtual na qualidade de vida em indivíduos parkinsonianos, a partir de pesquisas originais por meio de ensaios clínicos, ensaios clínicos randomizados, ensaios clínicos randomizados controlado, estudo piloto, ensaio clínico duplo-cego randomizado, estudo observacional prospectivo, ensaio clínico não controlado, sem restrição de faixa etária. Foram excluídos da amostra os artigos nas modalidades de revisão e estudos de casos, que aplicaram à RV em outras desordens neurológicas e que não especificaram o uso da RV na amostra selecionada.

\section{RESULTADOS e DISCUSSÃO}

$\mathrm{Na}$ primeira consulta feita, foram utilizadas as combinações dos seguintes descritores: "Realidade Virtual no Parkinson", em inglês "Reality Virtual at Parkinson", onde se obteve o resultado de 199 artigos encontrados, sendo apenas 4 artigos selecionados. Usando os descritores "Realidade Virtual, Parkinson, Qualidade de Vida" e "Reality Virtual, Parkinson, Qualidyof Life", foram encontrados 1.617 artigos, onde 15 foram selecionados. Com o descritor "Uso da Realidade Virtual no Parkinson", ou "Use of Virtual Reality in Parkinson", obteve-se o resultado de 17.300 artigos encontrados, o qual foram selecionados 30 estudos. Sobre o descritor "Efeitos do Parkinson na Qualidade de Vida realidade virtual e "Parkinson'seffectsonqualityoflife virtual reality", 2 foram encontrados e 2 selecionados. 
Foram encontrados cerca de 51 artigos que analisados enquadravam-se nos critérios de inclusão préestabelecidos, após a análise de inclusão, verificou-se os possíveis artigos que apresentaram os critérios de exclusão (Figura 1). Todos os artigos foram avaliados e selecionados respeitando os critérios descritos. Dentre os artigos achados, 3 destes se repetiram nas mesmas bases de dados. Conseguinte as leituras dos artigos, foram escolhidos aqueles que condizem com objetivo proposto no estudo e que tenham relação com a temática. A amostra final deste estudo foi composta por 7 artigos ${ }^{8,12,14-18}$.

Foi observado que a maioria dos artigos foram publicados na língua inglesa. Em questão aos anos de publicação, foram publicados: um artigo nos anos de 2013, 2015 e 2016 (14,28\%) e dois artigos nos anos de 2017 e $2018(28,57 \%)$.

Os tipos de estudos selecionados: um estudo de caso, seguindo para um estudo experimental do tipo ensaio clínico não controlado, um estudo clínico duplo-cego randomizado, acompanhando um estudo observacional prospectivo, um estudo piloto, seguindo para um ensaio clínico randomizado e um ensaio experimental controlado. Todos os estudos apresentaram um percentual de $11,11 \%$ cada (Tabela 1 ). Sobre os efeitos da realidade virtual na qualidade de vida dos pacientes parkinsonianos dois artigos relacionavam-se a melhora do equilíbrio, controle postural e marcha, dois direcionavam-se ao congelamento de 


\section{marcha, dois sobre o bem-estar emocional e um a respeito da realidade virtual para a cognição.}

Figura 1. Fluxograma contendo número de estudos e bases de dados dos artigos selecionados.

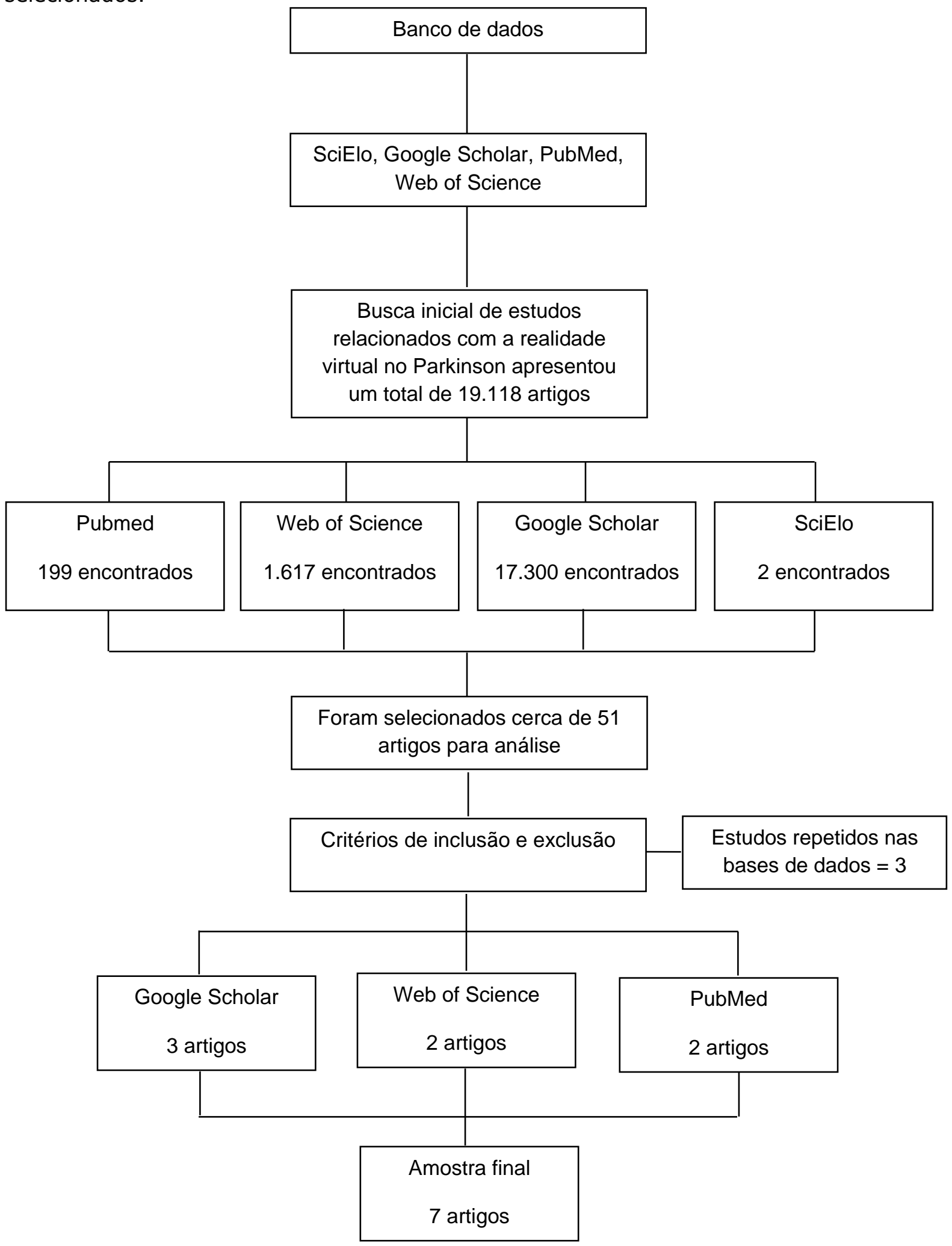


Tabela 1. Publicação quanto aos tipos de estudos.

\begin{tabular}{|c|c|c|}
\hline Tipos de estudos & $\begin{array}{c}\text { Publicações } \\
\left(\mathrm{n}^{\circ}\right)\end{array}$ & \begin{tabular}{c} 
Selecionadas \\
\hline Estudo de caso
\end{tabular} \\
\hline $\begin{array}{c}\text { Estudo experimental } \\
\text { do tipo ensaio clínico } \\
\text { não controlado }\end{array}$ & 1 & $11,11 \%$ \\
\hline $\begin{array}{c}\text { Estudo clínico duplo- } \\
\text { cego randomizado }\end{array}$ & 1 & $11,11 \%$ \\
\hline $\begin{array}{c}\text { Estudo observacional } \\
\text { prospectivo }\end{array}$ & 1 & $11,11 \%$ \\
\hline $\begin{array}{c}\text { Estudo piloto } \\
\text { Ensaio clínico } \\
\text { randomizado }\end{array}$ & 1 & $11,11 \%$ \\
\hline $\begin{array}{c}\text { Ensaio experimental } \\
\text { controlado }\end{array}$ & 1 & $11,11 \%$ \\
\hline
\end{tabular}

Sobre a amostra utilizada, em relação ao uso da RV e seus resultados, observa-se que os estudos utilizaram a RV como recurso para a melhora da qualidade de vida e prevenção das possíveis complicações futuras (Quadro 1).

Verificou-se que a maioria dos estudos analisados se relacionava a equilíbrio, seguido de cognição, congelamento de marcha e bem-estar emocional, todos com objetivos voltado para a melhora da QV desses indivíduos. Alguns estudos repetiram o mesmo tema ou correlacionavam-se a outros sintomas, sendo equilíbrio $(28,57 \%)$, congelamento de marcha $(28,57 \%)$, cognição $(14,28 \%)$, bem-estar emocional $(28,57 \%)$. 
Quadro 1. Classificação da análise dos estudos.

\begin{tabular}{|c|c|c|c|c|c|}
\hline $\mathbf{N}^{\circ}$ & $\begin{array}{c}\text { Desenho } \\
\text { Metodológico }\end{array}$ & $\begin{array}{c}\text { Amostra da } \\
\text { pesquisa }\end{array}$ & $\begin{array}{l}\text { Faixa } \\
\text { etária }\end{array}$ & Objetivos & Resultados obtidos \\
\hline $1^{18}$ & $\begin{array}{l}\text { Estudo de corte } \\
\text { observacional } \\
\text { prospectivo }\end{array}$ & $\begin{array}{c}16 \text { pacientes } \\
\text { de ambos os } \\
\text { sexos }\end{array}$ & $\begin{array}{c}18 \text { a } 82 \\
\text { anos }\end{array}$ & $\begin{array}{lr}\text { Avaliar } & \text { a } \\
\text { eficácia } & \text { dos } \\
\text { exercícios } & \text { de } \\
\text { equilíbrio } & \text { por } \\
\text { meios } & \text { de } \\
\text { jogos } & \text { de } \\
\text { realidade } & \\
\text { virtual na DP }\end{array}$ & 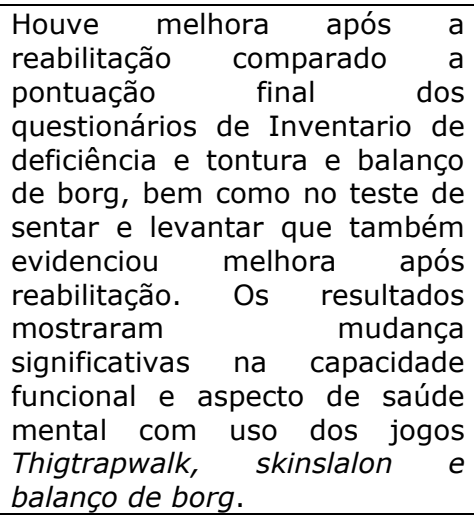 \\
\hline $2^{17}$ & Estudo piloto & $\begin{array}{c}10 \text { pacientes } \\
\text { de ambos os } \\
\text { sexos }\end{array}$ & 60 anos & $\begin{array}{l}\text { Determinar a } \\
\text { viabilidade e } \\
\text { aceitabilidade } \\
\text { da reabilitação } \\
\text { com um vídeo } \\
\text { game } \\
\text { personalizado } \\
\text { para tratar } \\
\text { distúrbios da } \\
\text { marcha e } \\
\text { equilíbrio em } \\
\text { pacientes com } \\
\text { DP e avaliar } \\
\text { seus efeitos } \\
\text { nos sinais } \\
\text { motores } \\
\text { incapacitantes. }\end{array}$ & $\begin{array}{l}\text { Após } 18 \text { sessões de } \\
\text { treinamento o questionário de } \\
\text { congelamento de marcha, } \\
\text { escala de marcha e equilíbrio e } \\
\text { pontuação axial diminuíram em } \\
39,38 \% \text { e } 41 \% \text {, } \\
\text { respectivamente. Houve um } \\
\text { aumento de } 35 \% \text { nas escalas } \\
\text { de confiança e equilíbrio } \\
\text { também. Os parâmetros } \\
\text { cinemáticos de marcha também } \\
\text { registraram melhora com } \\
\text { aumento do cumprimento de } \\
\text { passos e de velocidade da } \\
\text { marcha e diminuição o tempo } \\
\text { de apoio duplo. Os ganhos não } \\
\text { se mantiveram sem o } 0 \\
\text { treinamento constante, } \\
\text { perdendo-se após } 3 \text { meses de } \\
\text { intervenção. }\end{array}$ \\
\hline $3^{16}$ & $\begin{array}{l}\text { Ensaio clinico } \\
\text { randomizado }\end{array}$ & 73 pacientes & Adultos & $\begin{array}{lr}\text { Utilizar } & \text { um } \\
\text { padrão } & \text { de } \\
\text { marcha } & \text { de } \\
\text { realidade } & \\
\text { virtual } & \text { para } \\
\text { investigar } & \text { as } \\
\text { diferenças } & \\
\text { motoras. } & \end{array}$ & $\begin{array}{l}\text { Os pacientes com congelamento } \\
\text { da marcha demonstraram } \\
\text { aumento da velocidade motora } \\
\text { em comparação ao grupo não } \\
\text { congeladores e controles } \\
\text { principalmente em resposta a } \\
\text { gatilhos ambientalmente } \\
\text { salientes, incluindo } \\
\text { portas ( } p \text { 1/4 } 0,03 \text { e } 0,01 \\
\text { respectivamente) e a abertura } \\
\text { de uma porta deslizante ( } p 1 / 4 \\
0,036 \text { e } 0,048 \\
\text { respectivamente). }\end{array}$ \\
\hline $4^{8}$ & $\begin{array}{l}\text { Ensaio } \\
\text { experimental } \\
\text { controlado }\end{array}$ & $\begin{array}{c}\text { Pacientes } \\
\text { adultos com } \\
\text { DP }\end{array}$ & $\begin{array}{c}55 \text { a } 85 \\
\text { anos }\end{array}$ & $\begin{array}{l}\text { Testar se o } \\
\text { treinamento } \\
\text { de equilíbrio } \\
\text { com RV } \\
\text { baseado em } \\
\text { casa é mais } \\
\text { eficaz do que } \\
\text { o treinamento } \\
\text { de equilíbrio } \\
\text { doméstico } \\
\text { convencional } \\
\text { para melhorar } \\
\text { o equilíbrio, } \\
\text { caminhar, } \\
\text { e qualidade de } \\
\text { vida em } \\
\text { pacientes com } \\
\text { doença de } \\
\text { Parkinson } \\
\text { (DP). }\end{array}$ & $\begin{array}{l}\text { Após o treinamento, os } \\
\text { resultados mostraram queos } \\
\text { grupos tiveram melhor } \\
\text { desempenho na Escala de } \\
\text { Equilíbrio de Berg, Índice de } \\
\text { Marcha Dinâmica, teste Time } \\
\text { Upand Go cronometrado e } \\
\text { Questionário da Doença de } \\
\text { Parkinson no pós-teste e } \\
\text { acompanhamento do que no } \\
\text { pré-teste. As duas opções de } \\
\text { treinamentoforam igualmente } \\
\text { eficazes na melhoria do } \\
\text { equilíbrio, caminhada e } \\
\text { qualidade de vida. }\end{array}$ \\
\hline
\end{tabular}


Quadro 1 (cont.). Classificação da análise dos estudos.

\begin{tabular}{|c|c|c|c|c|c|}
\hline $\mathbf{N}^{\circ}$ & $\begin{array}{c}\text { Desenho } \\
\text { Metodológico }\end{array}$ & $\begin{array}{c}\text { Amostra da } \\
\text { pesquisa }\end{array}$ & $\begin{array}{l}\text { Faixa } \\
\text { etária }\end{array}$ & Objetivos & Resultados obtidos \\
\hline $5^{15}$ & Estudo de caso & $\begin{array}{c}11 \text { pacientes } \\
\text { no estágio I } \\
\text { e III na } \\
\text { escala de } \\
\text { Hoehn e } \\
\text { Yahr }\end{array}$ & $\begin{array}{l}\text { Idade } \\
\text { média de } \\
65 \text { anos }\end{array}$ & 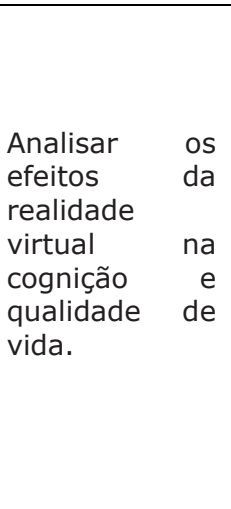 & $\begin{array}{l}\text { Os pacientes participaram de } 14 \\
\text { sessões onde praticaram quatro } \\
\text { jogos do Kinect. Todos foram } \\
\text { avaliados antes, durante e após } \\
\text { as intervenções, além de } 30 \\
\text { dias depois do tratamento. Os } \\
\text { resultados se mostraram } \\
\text { satisfatórios apenas no item } \\
\text { qualidade de vida que foi } \\
\text { avaliada pelo Questionário de } \\
\text { Doença de Parkinson (PDQ-39), } \\
\text { enquanto no item cognição } \\
\text { avaliado na escala Cognitiva de } \\
\text { Montreal (MoCA) não se obteve } \\
\text { mudanças significantes. }\end{array}$ \\
\hline $6^{12}$ & $\begin{array}{c}\text { Estudo } \\
\text { experimental, do } \\
\text { tipo ensaio clínico } \\
\text { não controlado }\end{array}$ & $\begin{array}{c}14 \text { pacientes } \\
\text { de ambos os } \\
\text { sexos }\end{array}$ & $\begin{array}{c}48 \text { e } 84 \\
\text { anos }\end{array}$ & $\begin{array}{lr}\text { Verificar } & \text { os } \\
\text { efeitos } & \text { do } \\
\text { tratamento } & \\
\text { com a RV } & \text { não } \\
\text { imersiva na } & \text { na } \\
\text { qualidade de } & \text { vida } \quad \text { de } \\
\text { pessoas com } \\
\text { doença de } \\
\text { Parkinson, } \\
\text { antes e } \\
\text { após } \\
\text { tratamento o } \\
\text { com aplicação } \\
\text { de } \\
\text { questionário } \\
\text { da doença de } \\
\text { Parkinson } \\
\text { (PDQ-39). }\end{array}$ & $\begin{array}{l}\text { Os pacientes foram avaliados } \\
\text { pela escala Questionário de } \\
\text { Doença deParkinson (PDQ-39) } \\
\text { antes das intervenções e } \\
\text { reavaliados ao final das } \\
\text { sessões. Os equipamentos } \\
\text { utilizados foram o Xbox 360, } \\
\text { Kinect, e os jogos Your Shape - } \\
\text { Fitness Envolved, Kinect } \\
\text { Adventures e Kinect Sports. Foi } \\
\text { utilizado o teste de Wilcoxon. } \\
\text { Houve uma diminuição em } \\
\text { áreas específicas para } \\
\text { mobilidade, bem-estar } \\
\text { emocional, estigma e cognição } \\
\text { e a pontuação do Questionário } \\
\text { da Doença de Parkinson-39 em } \\
\text { geral. }\end{array}$ \\
\hline $7^{14}$ & $\begin{array}{l}\text { Estudo clínico } \\
\text { duplo-cego } \\
\text { randomizado }\end{array}$ & 20 pacientes & $\begin{array}{l}50 \text { e } 80 \\
\text { anos }\end{array}$ & $\begin{array}{l}\text { Avaliar a } \\
\text { capacidade } \\
\text { funcional e a } \\
\text { QV indivíduos com } \\
\text { DP em RV com } \\
\text { o X-Box Kinect }\end{array}$ & $\begin{array}{l}\text { Os indivíduos foram avaliados } \\
\text { antes e após o tratamento com } \\
\text { a Escala Unificada de Avaliação } \\
\text { da Doença de Parkinson } \\
\text { (UPDS) e Doença de Parkinson } \\
\text { Questionário (PDQ-39) antes e } \\
\text { após intervenção, sendo } \\
\text { separados em dois grupos, } \\
\text { onde o controle foi tratado } \\
\text { apenas com terapia } \\
\text { convencional e o experimental } \\
\text { com RV e terapia convencional. } \\
\text { Houve uma redução nas } \\
\text { pontuações para todos os } \\
\text { domínios de UPDRS e PDQ-39. } \\
\text { Se destacando o emocional, } \\
\text { bem-estar, dificuldades sociais } \\
\text { da DP e desconforto. }\end{array}$ \\
\hline
\end{tabular}

\title{
Equilíbrio, cognição, congelamento de marcha e bem- estar emocional
}

\author{
Em um dos estudos, utilizaram um padrão de marcha
}

de realidade virtual para investigar as diferenças motoras

em pacientes com congelamento de marcha. Foram 
divididos grupos entre não congeladores e congeladores de marcha. Os pacientes tinham que navegar em um ambiente realista na tela com o uso de pedais para simular passos enquanto era avaliado sua capacidade de decidir e responder sinais associados a conflitos (congruente 'Vermelho', 'Verde' ou 'Azul') ou saliência ambiental (portas largas, estreitas e deslizantes). O tempo de passos foi usado como uma medida do rendimento motor. Os resultados se mostraram positivos em pacientes com congelamento da marcha, tendo aumento da resposta motora em comparação com o grupo não congeladores em controles e principalmente em resposta a gatilhos ambientalmente salientes, incluindo portas e abertura de uma deslizante ${ }^{14}$.

No estudo experimental controlado comparou-se 0 efeito do treinamento de equilíbrio com $\mathrm{RV}$ e treinamento de equilíbrio convencional na casa dos participantes. Foram selecionados vinte e três pacientes com DP idiopática, sendo submetidos a doze sessões de fisioterapia, de 50 minutos durante o período de treinamento de 6 semanas. 0 grupo experimental ( $n Z 11$ ) foi treinado com um sistema de treinamento de equilíbrio de realidade virtual feito sob medida, e o grupo de controle ( $n Z 12)$ foi treinado por um fisioterapeuta licenciado. Os resultados eram medidos durante as semanas, sendo classificadas em Semana 0 (pré-teste), Semana 6 (pós-teste) e Semana 8 (acompanhamento). Os grupos experimentais e controle demonstraram resultados iguais no pré-teste. Após o 
treinamento, ambos grupos tiveram melhor desempenho na Escala de Equilíbrio de Berg, Índice de Marcha Dinâmica, teste Time Up and Go cronometrado, e Questionário da Doença de Parkinson no pós-teste e acompanhamento, de que no pré-teste. Após análise dos dados, observou-se que não foram encontradas diferenças significativas entre os grupos com intervenção da RV e o grupo de acompanhamento convencional, pois os dois apresentaram os mesmos resultados ${ }^{12}$.

No estudo de corte observacional prospectivo, composto por 16 pacientes de ambos os sexos, avaliaram os efeitos da RV na DP. Os pacientes foram submetidos a uma serie de avaliações e testes aplicados antes e após a reabilitação. Para a reabilitação vestibular foi usado um equipamento Nintendo Wii, usando o wii-remote e wii balance bord, das quais são plataformas que servem para medir a força aplicada e que registra leves equilíbrios a medida em que ocorre mudanças de velocidade captadas por meio de sensores de pressão. Quatro jogos foram selecionados para serem aplicados (Soccer heading, inclinação da mesa, caminhada na corda bamba e skislalom). A reabilitação de equilíbrio corporal por meia da RV se mostrou eficiente na melhora de equilíbrio e capacidade funcional. Os jogos virtuais que mais mostraram eficácia foram Tightropewalk e Ski slalom ${ }^{8}$.

Para um estudo sobre os efeitos positivos que um jogo personalizado poderia trazer na reabilitação do congelamento de marcha e queda utilizaram um jogo que 
foi criado especificamente para a pesquisa realizada, que consistia na realização de movimentos rápidos de grande amplitude dos quatro membros feito pelo paciente. Foram selecionados para essa pesquisa dez pessoas com DP, submetidas ao programa de reabilitação por 18 sessões de treinamento no período de 6 semanas. Ao final das sessões os resultados indicaram que o jogo se mostrou viável para o uso em pacientes com DP, no entanto foi observado que os efeitos positivos no congelamento de marcha, equilíbrio, confiança e quedas que foram ganhos nas 18 sessões de tratamento, não foram mantidos após 3 meses da intervenção, por isso se faz necessário mais estudos controlados para manter os feitos positivos ${ }^{15}$.

Em um estudo experimental, do tipo ensaio clínico não controlado, utilizaram pacientes que se enquadravam nos estágios I a IV na escala de Hoen e Yahr com aplicação do questionário da doença de Parkinson (PDQ-39) antes e após o tratamento. Participaram da pesquisa 14 indivíduos, que passaram por 20 sessões de tratamento, 2 sessões semanais, com duração entre 30 e 40 minutos cada. Houve uma diminuição nos escores do Questionário da Doença de Parkinson-39 total e seus domínios quando comparados aos do período da avaliação e da reavaliação dos pacientes. Essa diminuição foi principalmente para os domínios mobilidade, bem-estar emocional, estigma e cognição, e o escore do Questionário da Doença de Parkinson-39 total16.

Um estudo clínico duplo cego randomizado avaliou a eficácia de uma reabilitação com a RV na capacidade 
funcional e QV dos pacientes. Foram utilizados 20 pacientes com idades entre 50 a 74 anos, separados em dois grupos controle e experimental, o grupo controle teve sessões com programas de exercícios e protocolos de atendimentos adaptados e o grupo experimental teve atendimento com RV aplicada a fisioterapia. A RV associada a fisioterapia se mostrou eficaz na QV dos pacientes, aumentando a motivação e interação deles, se destacando por envolver aspectos lúdicos e dinâmicos ${ }^{17}$.

Para um estudo de caso utilizaram onze pacientes com idade entre 65 anos, sendo classificados nos estádios I a III da Escala Hoehn e Yahr. Os pacientes tinham que jogar quatro jogos do Kinect Adventure durante 14 sessões com duração de 1 hora, duas vezes por semana em um período de sete semanas. Todos os pacientes passaram por avaliação antes, imediatamente após a intervenção e 30 dias após a intervenção. O item cognição foi avaliada por meio da Escala Cognitiva de Montreal (MoCA) e a QV foi avaliada por meio do Questionário de Doença de Parkinson (PDQ-39). Os resultados mostraram que somente as atividades do PDQ-39 do domínio da vida diária tiveram melhora estatisticamente significante, enquanto o escores da MoCA, em= geral, permaneceram os mesmos ${ }^{18}$.

Com relação ao objetivo do estudo, após leitura dos artigos selecionados para compor a amostra, observou-se que a RV aplicada a reabilitação do paciente é um aliado ao tratamento, proporcionando trabalho cognitivo, de marcha e equilíbrio, além de alguns estudos também relatarem 
melhora no bem-estar emocional. Apesar de apresentar resultados positivos, a RV deve ser aplicada constantemente para que se possa manter os ganhos obtidos.

\section{CONCLUSÃO}

Diante dos resultados expostos, a RV empregada como uma ferramenta de reabilitação em pacientes com DP se mostrou eficaz na melhora da QV deles, alcançando os domínios de mobilidade, bem-estar emocional, cognição, capacidade funcional, aumento do comprimento e velocidade do passo em congelamento de marcha, entre outros. No entanto, os resultados obtidos no decorrer das intervenções só se mantem permanentes se houver aplicabilidade constante do uso da RV, sendo necessário a produção de estudos científicos que enfoquem a durabilidade dos efeitos causados pela aplicação da RV na sintomatologia da DP.

\section{REFERÊNCIAS}

1.Hamani C, Lozano AM. Physiology and pathophysiology of Parkinson's disease. Ann NY AcadSci 2003;991:15-21. https://doi.org/10.1111/j.1749-6632.2003.tb07

2.Beitz JM. Parkinson's disease: a review. Front Biosci 2014;6:65-74. https://doi.org/10.2741/s415

3. Morris ME. Movement disorders in people with Parkinson disease: a model for physical therapy. Phys Ther 2000;80:578-97. https://pubmed.ncbi.nlm.nih.gov/10842411

4.Teive HAG. Etiopatogenia da doença de Parkinson. Rev Neurocienc 2005;13:201-14. https://doi.org/10.34024/rnc.2005.v13.8794

5.Keus SH, Bloem BR, Hendriks EJ, Bredero-Cohen AB, Munneke M. Evidence-based analysis of physical therapy in Parkinson's disease with recommendations for practice and research. Mov Disord 2007;22:451-60. https://doi.org/10.1002/mds.21244 
6.Lana RC, Álvares LMRS, Nasciutti-Pudente C, Goulart FRP, TeixeiraSalmela LF, et al. Percepção da qualidade de vida e indivíduos com doença de Parkinson através do PDQ-39. Ver Bras Fisioter 2007; 11:397-402.

https://doi.org/10.1590/S1413-

35552007000500011

7.Scattolin FAA. Qualidade de vida: a evolução do conceito e os instrumentos de medida. Ver Fac Cienc Med Sorocaba 2006;8:1-5.

https://revistas.pucsp.br/index.php/RFCMS/article/view/175/119

8.Severiano MIS, Zeigelboim BS, Teive HAG, Santos GJB, Fonseca VR. Effect of virtual reality in Parkinson's disease: a prospective observational study. Arq Neuropsiquiatr 2018;76:78-84. https://doi.org/10.1590/0004-282X20170195

9. Yen $\mathrm{CY}$, Lin $\mathrm{KH}$, Hu MH, Wu RM, Lu TW, Lin CH. Effects of virtual realityeaugmented balance training on sensory organization and attentional demand for postural control in people with Parkinson disease: a randomized controlled trial. Phys Ther 2011;91:862-74. https://doi.org/10.2522/ptj.20100050

10.Hsieh S, Lee CY, Tai CT. Set-shifting aptitude in Parkinson's disease: external versus internal cues. Psychol Rep 1995;77:339-49. https://doi.org/10.2466/pr0.1995.77.1.339

11. Qutubuddin AA, Pegg PO, Cifu DX, Brown R, McNamee S, Carne $W$. Validating the Berg Balance Scale for patients with Parkinson's disease: a key to rehabilitation evaluation. Arch Phys Med Rehabil 2005;86:789-92. https://doi.org/10.1016/j.apmr.2004.11.005

12.Yang WC, Wang HK, Wu RM, Lo CS, Lin KH. Home-based virtual reality balance training and conventional balance training in Parkinson's disease: A randomized controlled trial. J Formos Med Assoc 2016;115:734-43.

https://doi.org/10.1016/j.jfma.2015.07.012.

13.Cook DJ, Mulrow CD, Haynes RB. Systematic reviews: synthesis of best evidence for clinical decisions. Ann Intern Med 1997;126:37680. https://doi.org/10.7326/0003-4819-126-5-199703010-00006

14. Matar E, Shine JM, Naismith SL, Lewis SJG. Using virtual reality to explore the role of conflict resolution and environmental salience in Freezing of Gait in Parkinson's disease. Parkins Related Dis 2013;19:937-42. https://doi.org/10.1016/j.parkreldis.2013.06.002

15. Nuic D, Vinti M, Karachi C, Foulon P, Van Hamme A, Welter ML. The feasibility and positive effects of a customised videogame rehabilitation programme for freezing of gait and falls in Parkinson's disease patients: a pilot study. J Neuro Engin Rehab 2018;15:31-41. https://doi.org/10.1186/s12984-018-0375-x

16.Santana C, Lins O, Sanguinetti D, Silva F, Angelo T, Coriolano M, et al. Efeitos do tratamento com realidade virtual não imersiva na qualidade de vida de indivíduos com Parkinson. Rev Bras Geriatr Gerontol 2015;18:49-58. https://doi.org/10.1590/18099823.2015.14004

17.Fontoura V, Macêdo JGF, Silva LP. The role of rehabilitation with virtual reality in functional ability and quality of life of individuals with 
Parkinson's disease. Acta Fisiatr 2017;24:86-91. https://doi.org/10.5935/0104-7795.20170017

18.Souza M, Bacha JMR, Silva KG, Freitas TB, Torriani-Pasin C, Pompeu JE. Effects of virtual rehabilitation on cognition and quality of life of patients with Parkinson's disease. Fisioter Mov 2018;31:1-9. https://doi.org/10.1590/1980-5918.031.A012 